\title{
Some observations on SMON from Bombay
}

\author{
N. H. WADIA \\ From the Department of Neurology, J. J. Group of Hospitals, Byculla, Bombay, India
}

SUMMARY Nine patients in whom subacute myelo-opticoneuropathy (SMON) was diagnosed with varying degrees of confidence are discussed. The cases were discovered after a retrospective examination of our records for the period 1967-71, and a prospective search from March 1972 to date. Subacute myelopathy with predominant dysaesthesiae and greater involvement of the pyramidal tracts was seen more often than fully developed SMON. Subacute myelopathy was seen in six instances, opticomyelopathy in two and myeloneuropathy only once. Clioquinol could not be excluded as an aetiological agent. The difference in the reported prevalence of SMON between Japan and India is noted, and factors which may account for this difference are discussed. Problems related to the diagnosis of SMON outside Japan, and particularly in India, are stressed.

Subacute myelo-opticoneuropathy (SMON) as a clinical entity has only recently found its place in Western monographs and books on neurology (Pallis and Lewis, 1974; Ashworth, 1975; Le Quesne, 1975) although it is known to have afflicted the Japanese since 1956 (Sobue et al., 1971). A voluminous literature on all aspects of the disease has accumulated in Japan but there have been only occasional reports (often of single cases and at times of dubious authenticity) from Australia (Selby, 1972, 1973), India (Wadia, 1973), Switzerland (Kaeser and Wüthrich, 1970), Sweden (Osterman, 1971), Singapore (Tay, 1973), Great Britain (Terry, 1971; Spillane, 1971, 1972), United States (Kean, 1972), France (Grenier et al., 1972; Castaigne, et al., 1973), and Holland (Van Beeck and Scholten, 1972). The disproportionately high prevalence of this disease in Japan has been commented on by the Japanese themselves (Kono, 1971; Tsubaki et al., 1971; Shiraki, 1973). Tsubaki et al., (1971) were the first to point out that most of their patients had taken clioquinol before the onset of this disease. It soon became accepted, more so in Japan than elsewhere, that SMON was a clinically identifiable entity that could occur as a result of the ingestion of clioquinol.

An opportunity to acquire first hand knowledge of SMON was afforded by a visit to Japan in January 1972. It was later felt that a search for SMON in Bombay, a city in which clioquinol is ingested in vast amounts, might provide additional information. A

Accepted 28 October 1976 preliminary report was published concerning the possible occurrence of SMON in Bombay (Wadia1973).

This paper reports the cumulative data of two searches for cases of SMON, the first retrospective (based on clinical records and covering a five yeak period), the second prospective (covering a four and a half year period). These efforts resulted in the finding of nine patients considered, on clinicak. grounds, to be suffering from SMON. The main aim + of the surveys was to identify such patients, and to study them in depth with a view to excluding alternative diagnoses.

\section{Criteria for patient selection}

The criteria used to make a diagnosis of SMON were gathered from the Japanese literature and from the experience of examining such patients in Japan. Kono's (1971) diagnostic 'guideline' was liberally interpreted. Patients with neurological disorders of acute or subacute onset, characterised by persistent distal dysaesthesiae, myelopathy, peripheral neuropathy and optic atrophy, occurring in various combinations were considered possible cases of SMON. As in the Japanese cases, presence of all the symptoms and signs was not deemed necessary to make a diagnosis.

Kono (1971) stressed two cardinal symptoms, present in the overwhelming majority of the Japanese patients: (1) abdominal complaints preceding the neurological disorder, (2) acute or subacute dysaesthesiae, starting and predominating in the lower 
limbs. He also mentioned 'deep sensory disturbances' and weakness of the lower limbs. We therefore evaluated as possible cases of SMON all patients with persistent distal dysaesthesiae, especially if of ascending pattern and associated with evidence of myelopathy. Physical signs of associated peripheral or optic neuropathy were not considered essential. Although ankle jerks were absent in the majority of Japanese patients, this was not always the case. Their preservation, in our cases, was not held to invalidate a diagnosis of SMON. The first cardinal feature of the Japanese guidelines (namely abdominal disturbances or pain immediately preceding the onset of neurological symptoms) was not considered mandatory for diagnosis. We realised that if we accepted this criterion strictly, no case of SMON would be diagnosed.

After an overall assessment of the natural history, symptoms, and signs, a diagnosis of SMON was made on a descending scale of likelihood, the categories adopted being 'definite SMON', 'probable SMON', and 'compatible with SMON'. Several patients reviewed were deemed not to have SMON, though perhaps affected by a condition resembling it in some respects. The initial appraisal was made on purely clinical grounds, without reference to the question of clioquinol intake. On obtaining a history of clioquinol consumption its relation to the disease was assessed, taking into consideration the amount of drug ingested, the period over which it was taken, and the relationship of this period to the time of onset of symptoms. If the clioquinol intake had exceeded 10 grams and if the temporal relationship was close, it was felt that a diagnosis of clioquinol-induced SMON could not be excluded.

\section{Results}

The retrospective scrutiny was based on the inpatient records of the Department of Neurology at the J. J. Hospital, Bombay (covering the period 1967-71), and on the records for 1970-71 from my private practice; 5,168 records were examined with special attention to cases with a diagnosis of peripheral neuropathy, myelopathy, sensory ataxia, optic atrophy, and myeloneuropathy of known or unknown aetiology, or combinations of these. From this material, 83 records were thought to merit further study, and an attempt was made to trace and recall these patients. A satisfactory follow-up was possible in only 41 instances. The retrospective study yielded two cases $\left(\mathbf{R}_{1}\right.$ and $\left.\mathbf{R}_{2}\right)$ suffering from conditions considered 'compatible with SMON'. Clioquinol could not be excluded as the cause in the disorder. In nine further patients a somewhat similar neurological syndrome was seen: eight had not taken clioquinol and one had taken the drug only in very small amounts.

Prospectively, we kept a sharp look-out for SMON. Our interest in the condition was made known in professional or personal meetings with general physicians, gastroenterologists, and neurologists. Between March 1972 and August 1976 we saw seven patients $\left(P_{1}\right.$ to $\left.P_{7}\right)$ deemed to have SMON. All were questioned carefully for details of drug intake, with special reference to clioquinol.

The Table summarises the clinical manifestations, the electrophysiological findings, the clioquinol intake, and our overall conclusion regarding the nine patients accepted, with varying degrees of confidence, as suffering from SMON.

Table Clinical and electrophysiological data in SMON

\begin{tabular}{|c|c|c|c|c|c|c|c|c|c|}
\hline & \multicolumn{9}{|c|}{ Patients } \\
\hline & $\begin{array}{l}P_{1} \\
A F\end{array}$ & $\begin{array}{l}P_{2} \\
A B\end{array}$ & $\begin{array}{l}P_{3} \\
D F\end{array}$ & $\begin{array}{l}P_{5} \\
B K\end{array}$ & $\begin{array}{l}P_{6} \\
P P\end{array}$ & $\begin{array}{l}P_{7} \\
M B\end{array}$ & $\begin{array}{l}R_{1} \\
V D\end{array}$ & $\begin{array}{l}R_{2} \\
D J\end{array}$ & $\begin{array}{l}P_{4} \\
G D\end{array}$ \\
\hline Sex/age (yr) & $F / 60$ & $\mathrm{~F} / 60$ & $\mathbf{F} / 53$ & $\mathbf{M} / 55$ & $\mathbf{F} / 40$ & $F / 65$ & $\mathbf{M} / 21$ & $\mathrm{M} / 42$ & $\mathbf{F} / 9$ \\
\hline Preneurological abdominal disorder & + & - & - & - & - & \pm & - & - & - \\
\hline Pyramidal tract sign & + & $+t$ & ++ & + & - & + & ++ & $+t$ & + \\
\hline Posterior column disorder & + & + & + & + & ++ & ++ & + & + & + \\
\hline Ankle jerks & - & ++ & $+t$ & + & + & $+t$ & $+t$ & $+t$ & $+t$ \\
\hline Cutaneous sensory loss (level) & $\mathbf{L}_{1}$ & - & - & $\mathbf{L}_{3}$ & - & - & - & $\mathbf{D}_{10}$ & - \\
\hline Visual disturbance & - & + & - & + & - & - & - & - & - \\
\hline Bladder symptoms & + & - & - & + & - & - & + & + & - \\
\hline EMG-NC & $\downarrow$ & Normal & Normal & - & Normal & Normal & Normal & - & Normal \\
\hline \multicolumn{10}{|l|}{ Clioquinol intake } \\
\hline Amount in grams (approximate) & 25.2 & 1000 & 125 & 80 & 45 & 420 & 500 & 1000 & 21 \\
\hline Period & $32 \mathrm{~d}$ & $3 \mathrm{yr}$ & $2 \mathrm{yr}$ & $60 \mathrm{~d}$ & $4 \mathrm{mo}$ & $14 \mathrm{yr}$ & $4 \mathrm{yr}$ & $18 \mathrm{yr}$ & $42 \mathrm{~d}$ \\
\hline \multicolumn{10}{|l|}{ Improvement } \\
\hline $\begin{array}{l}\text { Drug continuing/drug stopped } \\
\text { Clinical pattern }\end{array}$ & $\frac{-1+}{\mathrm{MN}}$ & $\frac{-1+}{\mathrm{OM}}$ & $\frac{-1+}{M}$ & $\frac{-1+}{\mathrm{OM}}$ & $\frac{-1+}{\mathbf{M}}$ & $\frac{-1+}{M}$ & $\begin{array}{l}+1+ \\
\mathbf{M}\end{array}$ & $\begin{array}{l}+1+ \\
\mathbf{M}\end{array}$ & $\frac{-1+}{M}$ \\
\hline Final conclusion & D & $\mathbf{P}$ & $\mathbf{P}$ & $\mathbf{P}$ & $\mathbf{P}$ & $\mathbf{P}$ & $\mathrm{C}$ & $\mathrm{C}$ & $\mathrm{C}$ \\
\hline
\end{tabular}

EMG = electromyography. $\mathrm{NC}=$ nerve conduction study. $\downarrow=$ denervation and delayed nerve conduction. $+=$ present. $-=$ absent. $\mathrm{O}=$ optic neuropathy. $\mathbf{M}=$ myelopathy. $\mathbf{N}=$ peripheral neuropathy. $\mathbf{D}=$ definite $S M O N$. $P=$ probable $S M O N$. $C=$ compatible with $S M O N$. 
We considered one patient $\left(\mathrm{P}_{1}\right)$ as definitely suffering from SMON, and five $\left(P_{2}, P_{3}, P_{5}, P_{6}, P_{7}\right)$ as probably suffering from SMON. In three other patients $\left(\mathbf{R}_{1}, \mathbf{R}_{2}, \mathbf{P}_{4}\right)$ the natural history of the disease and the physical signs led us to consider the myelopathy as 'compatible with SMON'. Clioquinol could not be excluded as the cause of the disorder in these nine patients. Investigations (including myelography, CSF examination, and studies to exclude vitamin $\mathrm{B}_{12}$ deficiency) were carried out as required. No reasonable alternative diagnosis was forthcoming.

\section{DEFINITE SMON}

$\mathrm{AF}\left(\mathrm{P}_{1}\right)$, a 60 year old female, was admitted for surgery with constipation due to anal stenosis. She was at first prescribed clioquinol (Mexaform) $1.2 \mathrm{~g}$ daily and diloxanide furoate (Furamide) $0.5 \mathrm{~g}$ three times a day, for six days. Eleven days later both drugs were again prescribed, along with oxytetracycline for two weeks. On the ninth day of the second course she complained of abdominal discomfort. Three days later she experienced disturbing tingling, numbness, and weakness of the lower limbs with hesitancy of micturition. Severe paralysis of the lower limbs with dense sensory loss below T7 dermatome was noted. The drug was withdrawn and she gradually began to improve.

Two weeks later there was minimal residual weakness of the dorsiflexors and evertors of the feet, with slight hypertonia at the knees. The tendon reflexes were exaggerated except for the ankle jerks, which were absent. Both plantar responses were extensor. The patient complained of distal dysaesthesiae. Pain and tactile sense were impaired below the groins. Vibration and postural sense were diminished distally. Romberg's sign was positive and she could only walk with support.

Routine blood and CSF examinations were normal. The VDRL was negative. Electrodiagnostic examination revealed chronic partial denervation and slight slowing of motor and sensory nerve conduction in the lower limbs. Myelography showed no abnormality. She refused further investigation.

Seen again 18 months later, she had improved somewhat in that she walked without support. The ataxia and dysaesthesiae were still present. The reflexes were unchanged. She had no abdominal symptoms and had not taken further clioquinol.

In the face of other features of the condition, the absence of optic nerve involvement was not considered strong enough evidence, in this patient, to downgrade the rating from 'definite' to 'probable' SMON. There had been no diarrhoea or other abdominal disorder at any stage of the illness, except immediately before the onset of neurological symptoms.
PROBABLE SMON

The patients $\left(P_{2}, P_{3}, P_{5}, P_{6}, P_{7}\right)$ with a diagnosis of 'probable SMON' were given a lower rating because, though all of them had prolonged diarrhoea, none had preneurological abdominal disturbance or a definite peripheral neuropathy on clinical or electrophysiological assessment. Visual disturbances were complained of by two patients $\left(P_{2}\right.$ and $\left.P_{5}\right)$. Thorough investigations were carried out in three cases $\left(\mathbf{P}_{3}, \mathbf{P}_{6}\right.$, $P_{7}$ ) to exclude an alternative diagnosis. The other two patients $\left(\mathrm{P}_{2}, \mathrm{P}_{5}\right)$ did not permit a lumbar puncture or myelogram but, in the presence of visual symptoms and a subacute ascent of severe dysaesthesiae up to groin level, no alternative diagnosis seemed likely, although demyelinating disorders were considered. All patients had taken sufficient clioquinol, in an appropriate temporal relationship, to suggest that clioquinol might be a possible causative agent. Withdrawal of clioquinol arrested or reversed the progress of the disease.

The case history of $\mathbf{P}_{2}$ illustrates some of the differential diagnostic difficulties.

AB $\left(P_{2}\right)$, a 60 year old female with hypertension and osteoarthritis, had received many antibiotics ando $\omega$ other drugs for undiagnosed pyrexia of a month's duration. Towards the end of this period distressing paraesthesiae ascended within a few days to he $\mathbb{R}$ knees and thighs. The legs felt weak and she could only take a few steps with support.

Neurological examination carried out two and fout months later showed grade 2 hypertensive retinopathy but no optic atrophy. There was moderate spastic paraparesis with exaggerated deep reflexes and extensor plantar responses. Vibration and postural sense were impaired in the feet.

Five months later she complained of bilateral progressive dimness of vision. She could only count fingers at a distance of 1 metre and the visual fields were constricted. The fundi were unchanged.

Examination after a further three months revealed optic atrophy. Thereafter a slight lessening of the paraesthesiae and some improvement in walking were noticed. Examination still showed mild spastic weakness of the legs with only slight impairment in vibration and postural sense in the toes. Only now did she admit, on direct questioning, to having taken clioquinol (Mexaform) at a dose of $1200 \mathrm{mg}$ per day, for two and a half years before the onset of the neurological symptoms, for recurrent abdominal 'upsets' without diarrhoea. She had continued to take the tablets during the first four months of the illness, the drug only being withdrawn when visual disturbance appeared. Visual deterioration had then ceased and a slight improvement in walking and in the paraesthesiae was observed.

Electromyographic examination showed no evi- 
dence of a lower motor neurone lesion. Radiographs of the spine, blood and other routine examinations including VDRL were normal. CSF examination was not allowed.

The initial diagnosis had been of a subacute transverse myelitis. When optic atrophy appeared a demyelinating disorder of unknown aetiology was considered. Large doses of vitamin $\mathbf{B}_{12}$ had not arrested the condition. The massive dose of clioquinol ingested and the slight improvement following withdrawal of the drug led us to consider the case as 'probable SMON' due to clioquinol.

The case history of $P_{3}$ is recorded in an earlier paper (Case 3, Wadia, 1973).

NEUROLOGICAL DISORDER COMPATIBLE WITH SMON In three patients $\left(R_{1}, R_{2}\right.$, and $\left.P_{4}\right)$, a diagnosis of neurological disorder 'compatible with SMON' was made. The lower grading was given because these patients had neither immediate prodromal abdominal disturbance, nor peripheral or optic neuropathy. Clioquinol had been taken in large doses by $R_{1}$ and $\mathbf{R}_{2}$, though intermittently over a period of years. The condition had not worsened and had even improved slightly while the patients continued to take small but repeated doses of clioquinol. They were advised to stop the drug. The detailed case histories are recorded elsewhere (Case 1 and 2, Wadia, 1973). The history of $\mathrm{P}_{4}$ illustrates the difficulty in diagnosing SMON.

GD $\left(P_{4}\right)$, a girl aged nine years, had suffered from recurrent abdominal pain, sometimes associated with loose stools, for three years. She had taken several courses of metronidazole (Flagyl) and clioquinol (Mexaform) for Giardia lamblia infestation. From December 1974, she took metronidazole and clioquinol in two separate courses $(12 \mathrm{~g}$ of the former and $21 \mathrm{~g}$ of clioquinol, over a period of six weeks). Towards the end of the last course she began falling. Over the next few days she noticed increasing difficulty in walking and experienced bilateral paraesthesiae up to groin level. Clioquinol was stopped. Within 24 hours her gait began to improve. Two days later she was walking with a mild spastic ataxic gait. Postural sense was impaired in the toes and Romberg's sign was positive. There was slight weakness of dorsiflexion and eversion of the feet. The deep reflexes were present, including the ankle jerks which were, in fact, brisk. The plantar responses were flexor. Electromyography and nerve conduction velocity were within normal limits.

Had there been no history of clioquinol intake, this girl with dysaesthesiae and a fleeting spastic-ataxic myelopathy might have been diagnosed as suffering from transverse myelitis, possibly of viral origin. The story of clioquinol ingestion, in sufficient dose, before the onset of neurological symptoms em- boldened us to accept her condition as 'compatible with SMON'. She continues to remain well and takes neither metronidazole nor clioquinol. Her diarrhoea and other abdominal symptoms persist.

Accepting these nine patients as cases of SMON, analysis (Table) showed that females predominated $(6: 3)$, and that five of the nine patients were above the age of 50 years when first seen. All except $P_{1}$ were suffering from chronic, distressing, recurrent diarrhoea, flatulence, and abdominal discomfort or pain, often of many years standing. These symptoms were controlled or made tolerable by the frequent consumption of clioquinol and, in some instances, of other drugs. In only one or perhaps two patients were we convinced that there was a specific abdominal disturbance immediately preceding the neurological illness. Dysaesthesiae, usually up to the lower abdomen, were intense and distressing. They persisted distally, in all cases, despite withdrawal of clioquinol (except in patient $\mathbf{P}_{4}$ whose disease was fleeting).

Clinical evidence of posterior and/or lateral column involvement at a thoracolumbar level was present in all cases. Case $P_{6}$ had only sensory ataxia. One patient $\left(\mathrm{P}_{7}\right)$ had predominant sensory ataxia of spinal origin. Three patients $\left(\mathbf{P}_{1}, \mathbf{P}_{4}\right.$, and $\left.\mathbf{P}_{5}\right)$ had equal involvement of lateral and posterior columns. Four patients $\left(\mathbf{P}_{2}, \mathbf{P}_{3}, \mathbf{R}_{1}\right.$, and $\left.\mathbf{R}_{2}\right)$ had greater pyramidal tract involvement. The pyramidal tract disorder was usually evidenced as spasticity, weakness, and exaggerated deep reflexes; the plantar responses were extensor in only four of the seven patients with other evidence of lateral column involvement. Only one patient $\left(\mathbf{P}_{1}\right)$ was bedridden at the onset. The others were all ambulant. Even patient $P_{1}$ began walking after clioquinol withdrawal.

Surprisingly, peripheral neuropathy (as evidenced by absent ankle jerks, predominantly or restrictedly distal dysaesthesiae, and reduced nerve conduction velocities or distal denervation) was only seen unequivocally on one occasion $\left(\mathbf{P}_{1}\right)$.

Clioquinol had been taken in varying amounts by all patients. The maximum ingested was $1000 \mathrm{~g}$, taken continuously over a period of three years $\left(P_{2}\right)$. Most other patients had taken the drug repeatedly, over months or years. Clioquinol was actually being taken by all patients at the time of onset of their neurological complaints. No 'latent period' between stopping the drug and the onset of neurological symptoms was seen in any of our patients. Seven patients were deteriorating when we asked them to stop the drug: withdrawal of the drug was followed by arrest or improvement in their neurological condition. In two patients $\left(R_{1}\right.$ and $\mathbf{R}_{2}$ ) the inadvertent resumption of drug intake, admittedly in small doses, had not obviously resulted in deterioration. 
In summary, subacute myelopathy was seen in six patients $\left(\mathbf{P}_{\mathbf{3}}, \mathbf{P}_{\mathbf{4}}, \mathbf{P}_{\mathbf{6}}, \mathbf{P}_{\mathbf{7}}, \mathbf{R}_{\mathbf{1}}\right.$, and $\left.\mathbf{R}_{2}\right)$, opticomyelopathy in two patients $\left(P_{2}\right.$ and $\left.P_{5}\right)$, and myeloneuropathy once $\left(P_{1}\right)$. No case of the full syndrome (myelo-opticoneuropathy) was encountered. These nine cases are, in our opinion, acceptable as cases of SMON (of varying grades of certainty). No clear alternative diagnosis was evident. Clioquinol could not be excluded as the cause.

\section{Discussion}

It must be stressed at the outset that no attempt to carry out an epidemiological survey on the prevalence of SMON was made.

The points of interest centre around three main questions.

1. Are the cases described here those of SMON due to clioquinol, especially as no other published report has appeared from India, even after the common knowledge of SMON since 1971 ?

2. Why is SMON not more evident in India where so much clioquinol is consumed?

3. What are the problems encountered in diagnosing SMON?

When all the manifestations are present, including the preneurological abdominal disorder, SMON is an identifiable nosological entity with a narrow differential diagnosis. This is so even if there is no history of clioquinol intake. When the incidence of such cases assumes epidemic proportions, as reported from Japan, the diagnosis may become easier.

In our anxiety not to miss possible cases of SMON our criteria may have erred on the side of not being sufficiently strict. For instance, we included cases in whom some of the allegedly important components of SMON (such as preneurological abdominal disturbances and peripheral neuropathy) were absent. Hence it would not be difficult to challenge the diagnoses of 'probable SMON' or of 'compatible with SMON' in some of our cases. But for the same reasons we feel we have not underestimated the prevalence of SMON.

We felt emboldened to include for analysis cases which did not satisfy all the criteria of SMON, because despite the claim of the Japanese authors that this condition has distinctive features, a number of their own cases clearly did not fulfil all their own criteria. Sobue et al. (1971), while maintaining that myeloneuropathy with abdominal symptoms in Japan differs from any other neurological diseases reported in the literature, mention that the disease 'may be myelopathy or peripheral neuropathy or both, the combination varying according to the cases'. This leaves the door wide open for many unidentified peripheral neuropathies and myelo- pathies to be labelled SMON, merely on the basis of the patient having taken clioquinol. Kono (1971) quotes a figure of 2808 cases of 'suspected SMON' (as opposed to 5048 cases of 'confirmed SMON') in a national survey ending in October 1970. This suggests that it is not so easy to distinguish SMON from other diseases as is usually maintained. In a discussion of the differential diagnosis of SMON, Pallis (1976) stresses the difficulties, and maintains that large series tend to be 'contaminated' with patients suffering from other neurological disorders. Spillane (1971), Pallis and Lewis (1974), and Meade (1975) have expressed similar views.

If we accept our nine patients as cases of SMON, the following overall picture emerges. Females, specially elderly females, predominated. Abdominal symptoms (pain, diarrhoea, flatulence, and mucous discharge) are troublesome. This abdominal condition is nonspecific and was unrelated to amoebiasis. The gastroenterological symptomatology was relieved, often rapidly, by short or long courses of clioquinol and at times by other drugs. Abdominal pain or other abdominal symptoms, immediately preceding the nervous symptoms, were only seent once. Dysaesthesiae, often ascending up to the groins or umbilicus (with signs of myelopathy) weres complained of in all cases. Pyramidal tract signs were more obvious than ataxia, but both were often seen Surprisingly, definite peripheral neuropathy was onlyb confirmed once. Optic neuropathy was seen twice Clioquinol was being ingested in appropriate amounts right up to the day of onset of the neuros logical condition, unlike what was seen in some of the Japanese cases.

The widely held view, shared by Japanese authors (Kono, 1971; Tsubaki et al., 1971; Shiraki, 1973), that SMON is rare outside Japan appears to be confirmed by this study. Whereas some 10,000 cases of SMON were reported from Japan over a 15 year period, we have only been able to find nine cases in Bombay, in a little over nine years. If we consider the prospective study alone, we have seen only seven cases of SMON in four and a half years. It would be incorrect to compare the total Japanese experience, collated through a Commission of Inquiry, with the personal experience of an individual observer. Against this it might be mentioned: (a) that a nationwide scrutiny of retrospective data, carried out by a host of physicians, leads to a greater amount of observer bias, perhaps inflating the figures; (b) that no report of a series of cases, or even of single cases of SMON has appeared from India to date, despite common knowledge of the disorder through books and manufacturers' warnings on packages and leaflets. No reports of SMON have appeared from Brazil, Africa, Pakistan, Bangladesh, Thailand, 
Malaysia, and Indonesia, which between them must contain the bulk of the world's clioquinol-consuming population; (c) that the Japanese literature mentioned outbreaks and endemic pockets of SMON, even before clioquinol had been incriminated as a possible cause. We doubt that obvious clusters of cases, in and around big neurological centres in India, would have been missed, especially after the Japanese experience had been widely publicised. (d) Individual experiences in the two countries, may be worth comparing. J. J. Hospital and Bombay City can be roughly compared to Nagoya University Hospital and Nagoya City. Between 1956 and 1969 Sobue et al. (1971) were able to collect 752 cases of SMON (an average of 54 cases a year). In the subsequent two years, after Tsubaki et al. (1971) had propounded the clioquinol hypothesis, Sobue et al. (1973) added 349 further cases (an average of 175 cases per year). If such an epidemic had occurred in Bombay, especially during the four and a half years of our prospective study, they would have been detected. With a constant watch in a sizeable neurological practice, the contrast (seven cases in four and a half years versus 349 cases in two years) is too obvious to need further comment. It suggests that there is some factor in addition to clioquinol intake which operates in Japan. Many persons in India (Wadia, 1973) take more than ' 10 to $40 \mathrm{~g}$ of clioquinol', the dose deemed sufficient to cause SMON in Japan (Kono, 1971).

The factor determining this difference in prevalence has not yet been identified. Various conjectures have been made. Shiraki (1973) discusses fully the endogenous and exogenous factors, other than clioquinol, which could have contributed to the aetiology of SMON in Japan.

The possibility of racial or genetic predisposition (based on a presumed specific enzymatic defect in the Japanese leading to greater clioquinol neurotoxicity), has never been supported by any evidence. No definite case reports of Japanese overseas developing SMON have yet appeared (Meade, 1975). The greater chemical pollution of the Japanese environment may be a contributory factor, yet hard proof is lacking. The role of associated diseases, or of other drugs taken to treat them, needs evaluation. Many of the Japanese patients had tuberculosis, diabetes, malignancy, renal failure, etc. and must have taken drugs for them as did some of our patients. Drugs like metronidazole (Holdstock, 1975; Coxon and Pallis, 1976) and isonicotinic acid hydrazide are known to be neurotoxic, and on occasion to cause peripheral neuropathy. Could drug interaction have precipitated SMON in some patients, when each drug individually may have had no neurotoxic effect? Is it possible that associated diseases, especially renal or hepatic, contributed to SMON by impeding the excretion of clioquinol?

It is also possible, however, that no specific 'Japanese' factor exists. The difference in the reported prevalence of the disease would then reflect differences in the methods of gathering and interpreting data. SMON may still be misdiagnosed (or underdiagnosed) in India, but as Spillane (1971), Pallis and Lewis (1974), Meade (1975) and Pallis (1976) have pointed out it may also have been misdiagnosed (or overdiagnosed) in Japan.

How does one diagnose SMON, especially in its incomplete form, in the absence of any specific laboratory tests and without using the fact of clioquinol consumption as a diagnostic criterion? Today, the diagnosis of SMON outside Japan is largely, if not totally, based on an acceptance of the Japanese experience (Osterman, 1971; Selby, 1972). This results, at times, in the acceptance of patients as cases of SMON (or of their acceptance as cases of clioquinol neurotoxicity), when the neurological picture is far from typical. In such cases, the very fact of clioquinol intake tends to become-probably unconsciously-a diagnostic criterion. This is illustrated in the cases mentioned by Spillane (1971, 1972). Clioquinol is so widely consumed in India, that a history of clioquinol ingestion by patients with diseases of the CNS, including myelopathies of known and unknown aetiology, is not beyond the realm of mere coincidence. During the course of this study, and in the day-to-day practice of neurology, we have come across patients who resembled those diagnosed as SMON in this paper. They differed only in that most of them have not suffered preceding diarrhoea or other gastrointestinal disorder, and had, therefore, not taken clioquinol. Had they taken clioquinol, the diagnosis of SMON would have been seriously considered.

The difficulties in diagnosis are exemplified by the following examples.

1. We saw patients with slowly progressive spastic myelopathy who had taken clioquinol. SMON could not be diagnosed because of the insidiousness of the onset, the slowness of progression, and the rather indefinite nature of the paraesthesiae. No other cause for the chronic myelopathy could, however, be established.

2. We encountered patients with subacute myelopathy resembling SMON who had taken clioquinol, but for short times and in small doses. The temporal data made it unlikely that there was a cause and effect relationship.

3 . There were patients with fleeting transverse myelitis of uncertain aetiology who had casually taken small doses of clioquinol, within a short period of the onset of the symptoms. The clinical picture ruled out 
SMON and the dose ruled out a clioquinol-induced disorder. The association seemed coincidental. Our case $\mathrm{P}_{4}$ has been accepted as "compatible with SMON' because she took sufficient clioquinol, but the same objections could be levelled against the data presented as against the cases of Spillane (1971, 1972).

4. We have come across a patient the cause of whose myelopathy could not be found at the initial examination. He had taken clioquinol and SMON was considered. Later investigation established the correct diagnosis as myelopathy due to cervical spondylosis. If this patient had not been followed up or investigated carefully, a wrong diagnosis of clioquinol-induced SMON would have been made.

5. Finally, we have seen a patient with myeloneuropathy 'compatible with SMON' who had taken a fair amount of clioquinol, apparently in proper temporal relationship to the onset of disease. Yet on careful questioning it became apparent that one or two neurological symptoms had preceded the intake of clioquinol. Should this case have been included as an example of SMON? Even if the rest of the picture resembled SMON, can we justifiably attribute the condition to clioquinol?

These and other questions have arisen during the course of our observations, making us wonder about the validity of a diagnosis of neurotoxicity based merely on a history of preceding intake of a particular drug. In 1977, it would be imprudent totally to ignore the Japanese experience. If the factor which makes for the reported difference in SMON prevalence is genetic, SMON may never appear in India on an epidemic scale. But if the factor is environmental or infective, then a change in the Indian environment may result in the appearance of SMON. Meanwhile clioquinol has been recommended for production in bulk, on a priority basis, by a Parliamentary Committee in India (Report of the Committee on Drugs and Pharmaceutical Industry, 1975), on the grounds that it is an 'essential drug' and a 'household remedy'. However, it should be used with caution as all drugs should be, and more importantly, it should be withdrawn immediately should neurological symptoms develop.

A grant was made by Ciba (India) Ltd. to the Research Society, Grant Medical College, and J. J. Group of Hospitals, Bombay, to fund this project. Dr P. F. Irani performed the electrodiagnostic tests. I acknowledge their help gratefully.

\section{References}

Ashworth, B. (1975). Neuro-ophthalmology. In Recent
Advances in Clinical Neurology, p. 101. Edited by W. B. Matthews. Churchill Livingstone: London.

Castaigne, P., Rondot, P., Lenoel, Y., Duman, J.-L. R., and Autret, A. (1973). Myélopathie sévère, neuropathie periphérique et névrite optique survenues au cours d'un traitement par la chloroiodoquine (clioquinol). Thérapie, 28, 393-400.

Coxon, A., and Pallis, C. A. (1976). Metronidazole neuropathy. Journal of Neurology, Neurosurgery, and Psychiatry, 39, 403-405.

Grenier, B., Rolland, J. C., Casenave, C., and Desbuquois, G. (1972). Myéloradiculo-névrite subaiguë et traitement par la chloroiodoxyquinoleine. Archives Françaises de Pédiatrie, 29, 1015.

Holdstock, D. J. (1975). Drug treatment in Crohn's disease. Lancet, $2,1260$.

Kaeser, H. E., and Wüthrich, R. (1970). Zur Frage der Neurotoxizität der Oxychinolone. Deutsche Medizinische Wochenschrift, 95, 1685-1688.

Kean, B. H. (1972). Subacute myelo-optic neuropathy. Journal of the American Medical Association, 220, 243-244.

Kono, R. (1971). Subacute myelo-optico-neuropathy, a new neurological disease prevailing in Japan. Japanese Journal of Medical Science and Biology, 24, 195-216.

Le Quesne, P. M. (1975). Neurotoxic substances. In Modern Trends in Neurology-6, pp. 91-93. Edited by D. Williams. Butterworths: London.

Meade, T. W. (1975). Subacute myelo-optic neuropathy and clioquinol. An epidemiological case history for diagnosis. British Journal of Preventive and Sociat Medicine, 29, 157-169.

Osterman, P. O. (1971). Myelopathy after clioquinof treatment. Lancet, $2,544$.

Pallis, C. A. (1976). Proceedings Honolulu Symposium os 'Epidemiological issues in reported drug-induced illnesses? SMON and other examples'. McMaster University Press: Hamilton (Ontario).

Pallis, C. A., and Lewis, P. D. (1974). Neurological complications of clioquinol therapy. In The Neurology of Gastrointestinal Disease, pp. 179-188. Saunders: London.

Report of The Committee on Drugs and Pharmaceutical Industry. (1975). Ministry of Petroleum and Chemicals, Government of India. Chapter X, pp. 251-261. Government of India Press: New Delhi.

Selby, G. (1972). Subacute myelo-optic neuropathy in Australia. Lancet, 1, 123-125.

Selby, G. (1973). Subacute myelo-optic neuropathy (SMON). Neurotoxicity of clioquinols. Proceedings of the Australian Association of Neurologists, 9, 23-30.

Shiraki, H. (1973). Neuropathology of subacute myelooptic-neuropathy, 'SMON'. Neurology India, 20, Supplement 3, 395-419.

Sobue, I., Ando, K., Iida M., Takayanagi, T., Yamamura, Y., and Matsuoka, Y. (1971). Myelo-neuropathy with abdominal disorders in Japan. Neurology (Minneapolis), 21, 168-173.

Sobue, I., Ando, K., Iida, M., Takayanagi, T., Mukoyama, M., and Matsuoka, Y. (1973). Subacute myelo-opticoneuropathy in Japan. Neurology India, 20, Supplement $3,420-425$. 
Spillane, J. D. (1971). SMON. Lancet, 2, 1371-1372.

Spillane, J. D. (1972). SMON. Lancet, 1, 154.

Tay, C. H. (1973). SMON in Singapore. Lancet, 1, 1519.

Terry, S. I. (1971). Transient dysaesthesiae and persistent leucocytosis after clioquinol therapy. British Medical Journal, 3, 745.

Tsubaki, T., Honma, Y., and Hoshi, M. (1971). Neurological syndrome associated with clioquinol. Lancet, 1, 696-697.
Van Beeck, J. A., and Scholten, J. B. (1972). Polyneuropathie na langdurig gebruik van clioquinol (Enterovioform). Nederlands Tijdschrift voor Geneeskunde, 116 1621-1622.

Wadia, N. H. (1973). Is there SMON in India? Neurology India, 21, 95-103. 